\title{
ASYMPTOTIC BEHAVIOUR OF STOCHASTIC QUASI DISSIPATIVE SYSTEMS
}

\author{
Giuseppe Da Prato ${ }^{1}$
}

\begin{abstract}
We prove uniqueness of the invariant measure and the exponential convergence to equilibrium for a stochastic dissipative system whose drift is perturbed by a bounded function.
\end{abstract}

Mathematics Subject Classification. 47D07, 35K90.

Received January 8, 2002.

\section{INTRODUCTION}

Let us consider the following differential stochastic equation on a Hilbert space $H$ (with norm $|\cdot|$ and inner product $\langle\cdot, \cdot\rangle)$

$$
\left\{\begin{array}{l}
\mathrm{d} X(t)=(A X(t)+F(X(t))+G(X(t))) \mathrm{d} t+B \mathrm{~d} W(t) \\
X(0)=x \in H
\end{array}\right.
$$

where $A: D(A) \subset H \rightarrow H$ is self-adjoint and such that $A \leq-\omega I$ with $\omega>0, B: H \rightarrow H$ is linear bounded, $F: D(F) \subset H \rightarrow H$ is $m$-dissipative ${ }^{2}$ and $G: H \rightarrow H$ is Lipschitz continuous and bounded. Moreover $W(t)$ is a cylindrical Wiener process in $H$, defined in a probability space $(\Omega, \mathcal{F}, \mathbb{P})$.

System (1.1) is called quasi dissipative; it is called dissipative if $A+F+G$ is dissipative.

Assume that equation (1.1) has a solution $X(t, x)$. Then we consider the corresponding transition semigroup in $C_{b}(H)$ defined by the formula

$$
T_{t} \varphi(x):=\mathbb{E}[\varphi(X(t, x))], \quad x \in H, t>0, \varphi \in C_{b}(H)
$$

where $\mathbb{E}$ denotes the expectation. Here $C_{b}(H)$ is the Banach space of all uniformly continuous and bounded mappings $\varphi: H \rightarrow \mathbb{R}$ endowed with the norm $\|\varphi\|_{0}=\sup _{x \in H}|\varphi(x)|$.

Problem (1.1), which arises in several applications as: Reaction-Diffusion equations, Ginzburg-Landau models, Spin Systems, has been considered by several authors, see [12] and references therein and [4].

When system (1.1) is dissipative, one can show see [11], that for any $x \in H$ there exists the limit

$$
\lim _{t \rightarrow+\infty} \mathcal{L}(X(t, x))=\zeta
$$

Keywords and phrases: Stochastic systems, reaction-diffusion equations, invariant measures.

1 Scuola Normale Superiore di Pisa, piazza dei Cavalieri 7, 56126 Pisa, Italy; e-mail: DaPrato@sms.it

${ }^{2} F$ is called $m$-dissipative if $\langle F(x)-F(y), x-y\rangle \leq 0$ for all $x, y \in D(F)$ and the range of $I-F$ is equal to $H$. 
where $\mathcal{L}(X(t, x))$ is the law of $X(t, x)$ and $\zeta$ is the unique invariant measure of $T_{t}$, that is

$$
\int_{H} T_{t} \varphi(x) \zeta(\mathrm{d} x)=\int_{H} \varphi(x) \zeta(\mathrm{d} x), \quad t \geq 0, \varphi \in C_{b}(H) .
$$

As a consequence of (1.3) we have

$$
\lim _{t \rightarrow+\infty} T_{t} \varphi(x)=\int_{H} \varphi(y) \zeta(\mathrm{d} y), \quad x \in H, \varphi \in C_{b}(H),
$$

so that the invariant measure $\zeta$ is ergodic and strongly mixing.

When $G \neq 0$ system (1.1) is not necessarily dissipative, see Examples 1.2 and 1.3 below, and (1.3) does not hold in general. We notice that for a non dissipative system there is not in general existence and uniqueness of invariant measures.

In this paper we shall prove that, when $G$ is Lipschitz continuous and bounded and some suitable additional assumptions are fulfilled, there is an invariant measure $\zeta$ for (1.1) such that

$$
\left|T_{t} \varphi(x)-\int_{H} \varphi(y) \zeta(\mathrm{d} y)\right| \leq \kappa t^{-(1+\gamma) / 2} \mathrm{e}^{-\omega t}(1+|x|)\|\varphi\|_{0}, \quad x \in H, \varphi \in C_{b}(H),
$$

where $\kappa$ and $\gamma$ are positive constants.

An important consequence of (1.5) it that $\zeta$ is the unique invariant measure of $T_{t}$.

Remark 1.1. (i) If $G=0$ estimate (1.5) was proved in [11].

(ii) If $B$ has bounded inverse then the exponential convergence to equilibrium of $T_{t}$ was proved in [8] by a different method.

In the last part of the paper, we give an application of estimate (1.5) to the asymptotic behaviour, as $t \rightarrow \infty$, of the following Hamilton-Jacobi equation

$$
\left\{\begin{array}{l}
D_{t} u=\frac{1}{2} \operatorname{Tr}\left[B B^{*} D^{2} u\right]+\langle A x+F(x)+G(x), D u\rangle-\frac{1}{2}\left|B^{*} D u\right|^{2}, x \in D(A), \\
u(0)=\varphi \in C_{b}(H),
\end{array}\right.
$$

where $B^{*}$ is the adjoint of $B$. We show that

$$
\lim _{t \rightarrow+\infty} u(t, x)=-\log \int_{H} \mathrm{e}^{-\varphi(y)} \zeta(\mathrm{d} y), \quad x \in H .
$$

We end this section by giving two examples:

Example 1.2. Let us consider the following reaction-diffusion equation on $[0, \pi]$

$$
\left\{\begin{array}{l}
\mathrm{d} X(t, \xi)=\left[D_{\xi}^{2} X(t, \xi)+p(X(t, \xi))\right] \mathrm{d} t+\mathrm{d} W(t)(\xi), \quad \xi \in[0, \pi], t>0 \\
X(t, 0)=X(t, \pi)=0, \quad t>0 \\
X(0, \xi)=x(\xi), \quad \xi \in[0, \pi]
\end{array}\right.
$$

where $p$ is a polynomial having odd degree $s$ and negative leading coefficient, $x \in L^{2}(0, \pi)$ and $W$ is a cylindrical Wiener process in $H=L^{2}(0, \pi)$. 
Set $B=I$, and

$$
A x=D_{\xi}^{2}, \quad x \in D(A):=H^{2}([0, \pi]) \cap H_{0}^{1}([0, \pi]) .
$$

As easily checked, system (1.8) is dissipative if and only if $p^{\prime}(\xi)-\omega \leq 0$.

We show now, following [8], that there exist $F$ dissipative and $G$ Lipschitz continuous and bounded such that $p(\xi)=F(\xi)+G(\xi)$, so that (1.8) is of the form (1.1).

Let $\xi_{1}, \xi_{2} \in \mathbb{R}$ with $\xi_{1} \leq \xi_{2}$ be such that $p\left(\xi_{1}\right)=p\left(\xi_{2}\right)=0$ and $p$ is decreasing on $\left(-\infty, \xi_{1}\right] \cup\left[\xi_{2},+\infty\right)$. Then, setting

and

$$
F(\xi)=\left\{\begin{array}{l}
p(\xi) \text { if } \xi \in\left(-\infty, \xi_{1}\right] \cup\left[\xi_{2},+\infty\right), \\
0 \quad \text { if } \xi \in\left[\xi_{1}, \xi_{2}\right],
\end{array}\right.
$$

$$
G(x)=\left\{\begin{array}{l}
0 \quad \text { if } \xi \in\left(-\infty, \xi_{1}\right] \cup\left[\xi_{2},+\infty\right), \\
p(\xi) \quad \text { if } \xi \in\left[\xi_{1}, \xi_{2}\right]
\end{array}\right.
$$

we see that $F$ and $G$ have the required properties.

Example 1.3. Consider the following reaction-diffusion equation on $D:=[0, \pi]^{3}$.

$$
\left\{\begin{array}{l}
\mathrm{d} X(t, \xi)=\left[\Delta_{\xi} X(t, \xi)+p(X(t, \xi))\right] \mathrm{d} t+\left(-\Delta_{\xi}\right)^{-\delta / 2} \mathrm{~d} W(t)(\xi), \quad \xi \in D, t>0 \\
X(t, \xi)=0, \quad t>0, \quad \xi \in \partial D \\
X(0, \xi)=x(\xi), \quad \xi \in D
\end{array}\right.
$$

where $p$ is a polynomial having odd degree $s$ and negative leading coefficient, $\delta>0, x \in L^{2}(D)$ and $W$ is a cylindrical Wiener process in $H=L^{2}(D)$.

Set

$$
A x=\Delta_{\xi}, \quad x \in D(A):=H^{2}(D) \cap H_{0}^{1}(D) .
$$

Now, choosing $F$ and $G$ as in Example 1.2, we can write system (1.9) in the form (1.1) with $F$ dissipative and $G$ Lipschitz continuous and bounded.

\section{Hypotheses AND PRELIMINARIES}

In this section we recall some known results about problem (1.1).

Concerning the operators $A$ and $B$ we shall assume

\section{Hypothesis 2.1.}

(i) $A$ is a self-adjoint operator in $H$ such that

$$
\langle A x, x\rangle \leq-\omega|x|^{2}, \quad x \in H,
$$

for some $\omega>0$. Moreover $A^{-1}$ is compact.

(ii) $B \in L(H)^{3}$ and for any $t>0$ the linear operator $Q_{t}$, defined as

$$
Q_{t} x=\int_{0}^{t} \mathrm{e}^{s A} B B^{*} \mathrm{e}^{s A^{*}} x \mathrm{~d} s, \quad x \in H,
$$

is of trace class.

(iii) There exists $\rho \in\left(0, \frac{1}{2}\right)$ such that

$$
\int_{0}^{+\infty} s^{-2 \rho} \operatorname{Tr}\left[\mathrm{e}^{s A} B B^{*} \mathrm{e}^{s A^{*}}\right] \mathrm{d} s<+\infty .
$$

\footnotetext{
${ }^{3} L(H)$ represents the Banach algebra of all linear bounded operators from $H$ into $H$ endowed with the sup norm.
} 
We set

$$
Q_{t} x=\int_{0}^{t} \mathrm{e}^{s A} B B^{*} \mathrm{e}^{s A^{*}} x \mathrm{~d} s, \quad x \in H, \quad t \geq 0 .
$$

By Hypothesis 2.1 it follows that $Q_{t}$ is of trace class and that the stochastic convolution

$$
W_{A}(t)=\int_{0}^{t} \mathrm{e}^{s A} B B^{*} \mathrm{e}^{s A^{*}} \mathrm{~d} W(s), \quad t \geq 0,
$$

is a Gaussian random variable with mean 0 , covariance operator $Q_{t}$ and continuous paths, see [12].

We notice that the assumption that $A$ is self-adjoint is not essential, it could be replaced by $A$ variational.

Concerning $F$ we need two groups of assumptions, Hypotheses 2.2 and 2.3 below.

Hypothesis 2.2. $F$ is $m$-dissipative and $D(F)=K$, where $K$ is a reflexive Banach space continuously and densely embedded in $H$.

We denote by $A_{K}$ and $F_{K}$ the parts of $A$ and $F$ in $K$ :

$$
\begin{gathered}
D\left(A_{K}\right)=\{x \in D(A) \cap K: A x \in K\}, A_{K} x=A x, x \in D\left(A_{K}\right), \\
D\left(F_{K}\right)=\{x \in K: F(x) \in K\}, F_{K}(x)=F(x), x \in D\left(F_{K}\right) .
\end{gathered}
$$

The second group of assumptions is:

\section{Hypothesis 2.3.}

(i) $A_{K}: D\left(A_{K}\right) \subset K \rightarrow K$ generates a strongly continuous semigroup $\mathrm{e}^{t A_{K}}$ in $K$. Moreover, there exists $\omega_{1}>0$ such that

$$
\left\|\mathrm{e}^{t A_{K}}\right\|_{L(K)} \leq \mathrm{e}^{-\omega_{1} t}, t \geq 0
$$

(ii) $F_{K}$ is m-dissipative in $K$.

(iii) $F$ maps bounded subsets of $K$ into bounded subsets of $H$.

(iv) $W_{A}(t)$ takes values on $D\left(F_{K}\right)$ and

$$
\sup _{t \geq 0} \mathbb{E}\left(\left\|W_{A}(t)\right\|_{K}^{2}+\left\|F\left(W_{A}(t)\right)\right\|_{K}^{2}\right)<+\infty .
$$

We shall need two different notions of solutions of problem (1.1), mild solutions and generalized solutions.

Let $x \in K$ and $T>0$. We say that $X(\cdot)=X(\cdot, x)$ is a mild solution of $(1.1)$ on $[0, T]$ if

(i) $X(\cdot) \in C_{W}([0, T] ; H)^{4}$.

(ii) $X(t, x) \in K, \mathbb{P}$-a.s. for all $t \in[0, T]$ and

$$
X(t)=\mathrm{e}^{t A} x+\int_{0}^{t} \mathrm{e}^{(t-s) A}(F+G)(X(s)) \mathrm{d} s+W_{A}(t), \mathbb{P}-\text { a.s. }
$$

We say that $X(\cdot)=X(\cdot, x)$ is a generalized solution of $(1.1)$ on $[0, T]$ if assumption (i) holds and there exists a sequence $\left\{x_{n}\right\} \subset K$ convergent to $x$ in $H$, such that for any $n \in \mathbb{N}$ there exists a mild solution $X\left(\cdot, x_{n}\right)$ to $(1.1)$ on $[0, T]$ and

$$
\lim _{t \rightarrow+\infty} X\left(\cdot, x_{n}\right)=X(\cdot, x) \text { in } C_{W}([0, T] ; H) .
$$

The following result is proved in [11].

\footnotetext{
${ }^{4} C_{W}([0, T] ; H)$ is the Banach space of all continuous mappings $[0, T] \rightarrow L^{2}(\Omega, H)$ which are adapted to $W(t)$, endowed with the norm: $\|X\|_{C_{W}([0, T] ; H)}=\sup _{t \in[0, T]}\left[\mathbb{E}\left(|X(t)|^{2}\right)\right]^{1 / 2}$.
} 
Theorem 2.4. Assume that Hypotheses 2.1, 2.2, and 2.3, hold and that $G$ is Lipschitz continuous.

(i) If $x \in K$, problem (1.1) has a unique mild solution $X(\cdot, x)$.

(ii) If $x \in H$, problem (1.1) has a unique generalized solution $X(\cdot, x)$.

By the Markov property $T_{t}$ is a semigroup of linear bounded operators in $C_{b}(H)$. However, it is not strongly continuous in general. Following [3] we define the infinitesimal generator $S$ of $T_{t}$ through its Laplace transform $F(\lambda)$

$$
F(\lambda) f(x):=\int_{0}^{+\infty} \mathrm{e}^{-\lambda t} T_{t} f(x) \mathrm{d} t, \quad f \in C_{b}(H), \lambda>0 .
$$

It is easy to check that $F(\lambda)$ maps $C_{b}(H)$ into itself for all $\lambda>0$ and that $F(\lambda)$ is a pseudo-resolvent. Consequently, there exists a unique closed operator $S$ in $C_{b}(H)$ such that its resolvent $R(\lambda, S)$ is given by

$$
R(\lambda, S)=(\lambda-S)^{-1}=F(\lambda), \quad \lambda>0 .
$$

$S$ is called the infinitesimal generator of $T_{t}$ on $C_{b}(H)$.

Example 2.5. Let us consider equation (1.8). Define $A$ and $F$ as in Example 1.2. Then $A$ is self-adjoint and its spectrum $\sigma(A)$ is given by

Moreover

$$
\sigma(A)=\left\{-\pi^{2} k^{2}: k \in \mathbb{N}\right\}
$$

$$
Q_{t}=-\frac{1}{2} A^{-1}\left(I-\mathrm{e}^{2 t A}\right), t \geq 0
$$

Consequently

$$
\operatorname{Tr} Q_{t}=\sum_{k=1}^{\infty} \frac{1-\mathrm{e}^{-t k^{2}}}{k^{2}}<+\infty,
$$

and Assumptions 2.1(i, ii) are fulfilled. Also 2.1(iii) holds provided $\rho<1 / 4$.

Finally, the domain of $F$ is given by $K=L^{2 s}(0, \pi)$ and we have

$$
D\left(A_{K}\right)=H^{2,2 s}([0, \pi]) \cap H_{0}^{1,2 s}([0, \pi]), \quad D\left(F_{K}\right)=L^{2 s^{2}}([0, \pi]) .
$$

Now, it is not difficult to see [12] that also Hypotheses 2.2, and 2.3, are fulfilled and so Theorem 2.4 applies.

Example 2.6. Let us consider equation (1.9) and define $A, F$ and $B$ as in Example 1.3. Then $A$ is self-adjoint and its spectrum $\sigma(A)$ is given by

$$
\sigma(A)=\left\{-\pi^{2}\left(k_{1}^{2}+k_{2}^{2}+k_{3}^{2}\right):\left(k_{1}, k_{2}, k_{3}\right) \in \mathbb{N}^{3}\right\} .
$$

Moreover

Consequently

$$
Q_{t}=\frac{1}{2}(-A)^{-1-\delta}\left(I-\mathrm{e}^{2 t A}\right), t \geq 0
$$

$$
\operatorname{Tr} Q_{t}=\sum_{k=1}^{\infty} \frac{1-\mathrm{e}^{-t\left(\left(k_{1}^{2}+k_{2}^{2}+k_{3}^{2}\right)\right.}}{\left(k_{1}^{2}+k_{2}^{2}+k_{3}^{2}\right)^{1+\delta}}<+\infty,
$$

provided $\delta>1 / 2$. Therefore Hypotheses $2.1(\mathrm{i}$, ii) is fulfilled. Moreover, choosing $\rho<\min \{1 / 2,2(\delta-1 / 2)\}$, also Hypothesis 2.1(iii) holds.

Finally, the domain of $F$ is given by $K=L^{2 s}(D)$ and we have

$$
D\left(A_{K}\right)=H^{2,2 s}(D) \cap H_{0}^{1,2 s}(D), \quad D\left(F_{K}\right)=L^{2 s^{2}}(D) .
$$

Then, it is easy to see that Hypotheses 2.2, and 2.3, are fulfilled. Therefore, if $\delta>1 / 2$, Theorem 2.4 applies. 


\section{The MAIn RESUlt}

In this section we shall assume that Hypotheses 2.1, 2.2, and 2.3, hold.

\subsection{Existence of an invariant measure}

We first prove two estimates.

Lemma 3.1. Assume that Hypotheses 2.1, 2.2, and 2.3, hold.

(i) Let $x \in H$ and let $X(t, x)$ be the generalized solution of (1.1). Then there exists $\kappa_{1}>0$ such that

$$
\mathbb{E}|X(t, x)|^{2} \leq \kappa_{1}\left(1+\mathrm{e}^{-2 \omega t}|x|^{2}\right), \quad t \geq 0
$$

(ii) Let $x \in K$ and let $X(t, x)$ be the mild solution of (1.1). Then there exists a constant $\kappa_{2}>0$ such that

$$
\mathbb{E} \mid F\left(\left.X(t, x)\right|^{2} \leq \kappa_{1}\left(1+\mathrm{e}^{-2 \omega t}|x|_{K}^{2}\right), \quad t \geq 0 .\right.
$$

Proof. Let us prove (i). Setting $Z(t)=X(t, x)-W_{A}(t)$, equation (1.1) becomes

$$
\left\{\begin{array}{l}
\frac{\mathrm{d}}{\mathrm{d} t} Z(t)=A Z(t)+F\left(Z(t)+W_{A}(t)\right)+G\left(Z(t)+W_{A}(t)\right), \\
Z(0)=x
\end{array}\right.
$$

Multiplying the first equation in (3.3) by $Z(t)$ and taking into account $(2.1)$ and the dissipativity of $F$, we obtain

$$
\begin{aligned}
\frac{1}{2} \frac{\mathrm{d}}{\mathrm{d} t}|Z(t)|^{2} & \leq-\omega|Z(t)|^{2}+\left\langle F\left(Z(t)+W_{A}(t)\right)-F\left(W_{A}(t)\right), Z(t)\right\rangle+\left\langle F\left(W_{A}\right), Z(t)\right\rangle+\|G\|_{0}|Z(t)| \\
& \leq-\omega|Z(t)|^{2}+\left\langle F\left(W_{A}\right), Z(t)\right\rangle+\|G\|_{0}|Z(t)| \\
& \leq-\frac{\omega}{2}|Z(t)|^{2}+c_{1}\left(\left|F\left(W_{A}\right)\right|^{2}+1\right)
\end{aligned}
$$

where $c_{1}$ is a suitable constant.

By the Gronwall lemma it follows that

$$
|Z(t)|^{2} \leq \mathrm{e}^{-\omega t}|x|^{2}+2 c_{1} \int_{0}^{t} \mathrm{e}^{-2 \omega(t-s)}\left(\left|F\left(W_{A}(s)\right)\right|^{2}+1\right) \mathrm{d} s
$$

and finally, for some positive constant $c_{2}$

$$
|X(t, x)|^{2} \leq c_{2} \mathrm{e}^{-2 \omega t}|x|^{2}+c_{2}\left(\int_{0}^{t} \mathrm{e}^{-2 \omega(t-s)}\left(\left|F\left(W_{A}(s)\right)\right|^{2}+1\right) \mathrm{d} s+\left|W_{A}(t)\right|^{2}\right) .
$$

Now (3.1) follows taking expectation and recalling (2.5).

Let us now prove (ii). We have

$$
\frac{\mathrm{d}^{+}}{\mathrm{d} t}|Z(t)|_{K} \leq-\omega_{1}|Z(t)|_{K}+\|G\|_{0}+\left\langle F\left(Z(t)+W_{A}(t)\right), \nu_{t}\right\rangle
$$


where $\frac{\mathrm{d}^{+}}{\mathrm{d} t}$ represents the right derivative and $\nu_{t}$ belongs to the subdifferential of $|Z(t)|_{K}$, see [17]. Since $F$ is dissipative in $K$ (by Hypothesis 2.3(ii)), we obtain

$$
\begin{aligned}
\frac{\mathrm{d}^{+}}{\mathrm{d} t}|Z(t)|_{K} & \leq-\omega_{1}|Z(t)|_{K}+\|G\|_{0}+\left\langle F\left(Z(t)+W_{A}(t)\right)-F\left(W_{A}(t)\right), \nu_{t}\right\rangle+\left\langle F\left(W_{A}(t)\right), \nu_{t}\right\rangle \\
& \leq-\omega_{1}|Z(t)|_{K}+\|G\|_{0}+\left|F\left(W_{A}(t)\right)\right|_{K} .
\end{aligned}
$$

Now the conclusion follows using the Gronwall lemma and taking into account (2.5).

We are now in position to prove the existence of an invariant measure for $T_{t}$. To this purpose let us first recall that there exists a positive constant $\kappa_{\rho}$ such that

$$
\left|(-A)^{\rho} \mathrm{e}^{t A} x\right| \leq \kappa_{\rho} t^{-\rho} \mathrm{e}^{-\omega t}|x|, x \in H, t>0
$$

where $\rho$ was defined in $(2.2)$.

Proposition 3.2. Assume that Hypotheses 2.1, 2.2, and 2.3, hold. Then there exists an invariant measure $\zeta$ for $T_{t}$. Moreover

$$
\int_{H}|x|^{2} \zeta(\mathrm{d} x)<+\infty
$$

Proof. Let $x \in K$ and let $X(t, x)$ be the mild solution of (1.1). Then we have

$$
\begin{gathered}
(-A)^{\rho} X(t, x)=(-A)^{\rho} \mathrm{e}^{t A} x \\
+\int_{0}^{t}(-A)^{\rho} \mathrm{e}^{(t-s) A}(F+G)(X(s, x)) \mathrm{d} s \\
+(-A)^{\rho} W_{A}(t), \quad t \geq 0, x \in H .
\end{gathered}
$$

Moreover

$$
\begin{aligned}
\mathbb{E}\left[\left|(-A)^{\rho} W_{A}(t)\right|^{2}\right] & =\int_{0}^{t} \operatorname{Tr}\left[(-A)^{\rho} \mathrm{e}^{s A} B B^{*}\left(-A^{*}\right)^{\rho} \mathrm{e}^{s A^{*}}\right] \\
& \leq 2 \kappa_{\rho}^{2} \int_{0}^{t} t^{-2 \rho} \operatorname{Tr}\left[\mathrm{e}^{s A} B B^{*} \mathrm{e}^{s A^{*}}\right] \mathrm{d} s
\end{aligned}
$$

Therefore, in view of Hypothesis 2.1(iii), there exists $c_{\rho}>0$ such that

$$
\mathbb{E}\left[\left|(-A)^{\rho} W_{A}(t)\right|^{2}\right] \leq c_{\rho}, \quad t \geq 0 .
$$

From (3.6) and (3.7) it follows that there exists a constant $c_{1, \rho}(x)$ such that

$$
\mathbb{E}\left|(-A)^{\rho} X(t, x)\right| \leq t^{-\rho} c_{1, \rho}(x), \quad t \geq 0 .
$$

We can now show that the set of the laws of $X(t, x),\{\mathcal{L}(X(t, x))\}_{t \geq 1}$, is tight. For any $R>0$, denote by $B_{R}^{\rho}$ the ball $B_{R}^{\rho}:=\left\{y \in H:|y|_{D\left((-A)^{\rho}\right)}<R\right\}$. Then we have

$$
\begin{aligned}
\mathcal{L}(X(t, x))\left(\left(B_{R}^{\beta}\right)^{c}\right)= & \int_{|y|_{D\left((-A)^{\rho} \geq R\right.}} \mathcal{L}(X(t, x))(\mathrm{d} y) \\
& \leq \frac{1}{R} \int_{H}|y|_{D\left((-A)^{\rho}\right)}|\mathcal{L}(X(t, x))(\mathrm{d} y)=\mathbb{E}|(-A)^{\rho} X(t, x) \mid \leq \frac{1}{R} c_{1, \rho}(x) .
\end{aligned}
$$


This implies tightness of $\{\mathcal{L}(X(t, x))\}_{t \geq 1}$, because the embedding $D\left((-A)^{\rho}\right) \subset H$ is compact by Hypothesis 2.1(i). Therefore, from the Krylov-Bogoliubov theorem it follows that there exists an invariant measure $\zeta$ for $T_{t}$.

Let us prove now (3.5). By (3.1) we have in fact, integrating with respect to $\zeta$ and taking into account the invariance of $\zeta$

Choosing $t_{0}>0$ such that $\kappa_{1} \mathrm{e}^{-2 \omega t_{0}}<1$ we have

$$
\int_{H}|x|^{2} \zeta(\mathrm{d} x) \leq \kappa_{1}\left(1+\mathrm{e}^{-2 \omega t} \int_{H}|x|^{2} \zeta(\mathrm{d} x)\right)
$$

$$
\int_{H}|x|^{2} \zeta(\mathrm{d} x) \leq \frac{\kappa_{1}}{1-\kappa_{1} \mathrm{e}^{-2 \omega t_{0}}}
$$

and (3.5) is proved.

\subsection{Strong Feller property}

Together with problem (1.1) it is useful to consider the following dissipative problem, obtained by setting $G=0$ in $(1.1)$

$$
\left\{\begin{array}{l}
\mathrm{d} Y(t)=(A Y(t)+F(Y(t))) \mathrm{d} t+B \mathrm{~d} W(t) \\
Y(0)=x \in H
\end{array}\right.
$$

Again by Theorem 2.4 problem (3.8) has a unique generalized solution $Y(t, x)$ for any $x \in H$. We denote by $P_{t}$ the corresponding transition semigroup

$$
P_{t} \varphi(x):=\mathbb{E}[\varphi(Y(t, x))], \quad x \in H, \varphi \in C_{b}(H),
$$

and by $N$ its infinitesimal generator in $C_{b}(H)$, defined through its resolvent.

We also need to introduce the Yosida approximations of $F$. For any $\alpha>0$ we set

$$
F_{\alpha}(x):=\frac{1}{\alpha}\left(J_{\alpha}(x)-x\right), \quad x \in H
$$

where

$$
J_{\alpha}(x):=(I-\alpha F)^{-1}(x), \quad x \in H, \alpha>0 .
$$

$F_{\alpha}$ is Lipschitz continuous, but not differentiable in general. Therefore we introduce a further regularization, as in [10], by setting

$$
F_{\alpha, \beta}(x):=\int_{H} \mathrm{e}^{\beta C} F_{\alpha}\left(\mathrm{e}^{\beta C} x+y\right) N_{\frac{1}{2} C^{-1}\left(\mathrm{e}^{2 \beta C}-1\right)}(\mathrm{d} y), \quad \alpha, \beta>0,
$$

where $C: D(C) \subset H \rightarrow H$ is a self-adjoint negative definite operator such that $C^{-1}$ is of trace class and $N_{\frac{1}{2}} C^{-1}\left(\mathrm{e}^{2 \beta C}-1\right)$ is the Gaussian measure with mean 0 and covariance operator $\frac{1}{2} C^{-1}\left(\mathrm{e}^{2 \beta C}-1\right)$.

${ }^{2} F_{\alpha, \beta}$ is dissipative and of class $C^{\infty}$ with bounded derivatives of all orders. Moreover, as $\alpha, \beta \rightarrow 0, F_{\alpha, \beta} \rightarrow F$ pointwise, see [12].

Now we consider, for any $\alpha>0, \beta>0$, the approximating problems

$$
\left\{\begin{array}{l}
\mathrm{d} X_{\alpha, \beta}(t)=\left[A X_{\alpha, \beta}(t)+\left(F_{\alpha, \beta}+G\right)\left(X_{\alpha, \beta}(t)\right] \mathrm{d} t+B \mathrm{~d} W(t),\right. \\
X_{\alpha, \beta}(0)=x \in H
\end{array}\right.
$$


and

$$
\left\{\begin{array}{l}
\mathrm{d} Y_{\alpha, \beta}(t)=\left(A Y_{\alpha, \beta}(t)+F_{\alpha, \beta}\left(Y_{\alpha, \beta}(t)\right) \mathrm{d} t+B \mathrm{~d} W(t),\right. \\
Y_{\alpha, \beta}(0)=x \in H .
\end{array}\right.
$$

We denote by $X_{\alpha, \beta}(t, x)$ and $Y_{\alpha, \beta}(t, x)$ the mild solutions of (3.11) and (3.12) respectively. It is easy to check, arguing as in [7], that for any $x \in H$ and $T>0$ we have,

$$
\lim _{\alpha \rightarrow 0, \beta \rightarrow 0} X_{\alpha, \beta}(\cdot, x)=X(\cdot, x) \quad \text { in } C_{W}(0, T ; H),
$$

and

$$
\lim _{\alpha \rightarrow 0, \beta \rightarrow 0} Y_{\alpha, \beta}(\cdot, x)=Y(\cdot, x) \quad \text { in } C_{W}(0, T ; H),
$$

where $X(\cdot, x)$ and $Y(\cdot, x)$ are the generalized solutions of (1.1) and (3.8) respectively.

We shall denote by $T_{t}^{\alpha, \beta}$ and $P_{t}^{\alpha, \beta}$ the transition semigroups

$$
\begin{aligned}
& T_{t}^{\alpha, \beta} \varphi(x)=\mathbb{E}\left[\varphi\left(X_{\alpha, \beta}(t, x)\right)\right], t \geq 0, \varphi \in C_{b}(H), \\
& P_{t}^{\alpha, \beta} \varphi(x)=\mathbb{E}\left[\varphi\left(Y_{\alpha, \beta}(t, x)\right)\right], t \geq 0, \varphi \in C_{b}(H),
\end{aligned}
$$

respectively, and by $S_{\alpha, \beta}$ and $N_{\alpha, \beta}$ their infinitesimal generators in $C_{b}(H)$.

Since $F_{\alpha, \beta}$ is regular, it is easy to see that $Y_{\alpha, \beta}(t, x)$ is Gateaux differentiable with respect to $x$ and setting $\eta_{\alpha, \beta}^{h}(t, x)=D Y_{\alpha, \beta}(t, x)$ we have

$$
\left\{\begin{array}{l}
D_{t} \eta_{\alpha, \beta}^{h}(t, x)=A \eta_{\alpha, \beta}^{h}(t, x)+D F_{\alpha, \beta}\left(X_{\alpha, \beta}(t, x)\right) \eta_{\alpha, \beta}^{h}(t, x), \\
\eta_{\alpha, \beta}^{h}(0, x)=h
\end{array}\right.
$$

The following result will be useful later.

Proposition 3.3. Assume that Hypotheses 2.1, 2.2 and 2.3 hold. Let $h \in H$ and let $\eta_{\alpha, \beta}^{h}$ be the solution of (3.16). Then for any $\gamma \in[0,1]$ we have

$$
\int_{0}^{T}\left|(-A)^{\gamma / 2} \eta_{\alpha, \beta}^{h}(t, x)\right|^{2} \mathrm{~d} t \leq 2^{-\gamma} T^{1-\gamma}|h|^{2}, \quad T>0 .
$$

Proof. We first notice that, since $F_{\alpha, \beta}$ is dissipative, we have

$$
\left\langle D F_{\alpha, \beta}(y) z, z\right\rangle \leq 0, \quad y, z \in H .
$$

Therefore, multiplying the first equation in (3.16) by $\eta_{\alpha, \beta}^{h}(t, x)$, yields

$$
\frac{1}{2} \frac{\mathrm{d}}{\mathrm{d} t}\left|\eta_{\alpha, \beta}^{h}(t, x)\right|^{2} \leq\left\langle A \eta_{\alpha, \beta}^{h}(t, x), \eta_{\alpha, \beta}^{h}(t, x)\right\rangle=-\left|(-A)^{1 / 2} \eta_{\alpha, \beta}^{h}(t, x)\right|^{2},
$$

for $x \in H$, and $t \geq 0$. It follows

$$
\frac{1}{2} \frac{\mathrm{d}}{\mathrm{d} t}\left|\eta_{\alpha, \beta}^{h}(t, x)\right|^{2} \leq-\omega\left|\eta_{\alpha, \beta}^{h}(t, x)\right|^{2}, \quad x \in H, t \geq 0,
$$


which yields, by a standard comparison result,

$$
\left|\eta_{\alpha, \beta}^{h}(t, x)\right|^{2} \leq \mathrm{e}^{-2 \omega t}|h|^{2}, \quad x \in H, t \geq 0 .
$$

Now, by (3.18) we find for any $T>0$

$$
\frac{1}{2}\left|\eta_{\alpha, \beta}^{h}(T, x)\right|^{2}+\int_{0}^{T}\left|(-A)^{1 / 2} \eta_{\alpha, \beta}^{h}(t, x)\right|^{2} \mathrm{~d} t \leq \frac{1}{2}|h|^{2},
$$

and consequently

$$
\int_{0}^{T}\left|(-A)^{1 / 2} \eta_{\alpha, \beta}^{h}(t, x)\right|^{2} \mathrm{~d} t \leq \frac{1}{2}|h|^{2} .
$$

By using the well known interpolatory estimate

$$
\left|(-A)^{\gamma / 2} x\right| \leq|x|^{1-\gamma}\left|(-A)^{1 / 2} x\right|^{\gamma}, \quad x \in D\left((-A)^{1 / 2}\right), \gamma \in[0,1],
$$

we find, using the Hölder inequality,

$$
\begin{aligned}
& \int_{0}^{T}\left|(-A)^{\gamma / 2} \eta_{\alpha, \beta}^{h}(t, x)\right|^{2} \mathrm{~d} t \leq \int_{0}^{T}\left|\eta_{\alpha, \beta}^{h}(t, x)\right|^{2(1-\gamma)}\left|(-A)^{1 / 2} \eta_{\alpha, \beta}^{h}(t, x)\right|^{2 \gamma} \mathrm{d} t \\
& \leq\left(\int_{0}^{T}\left|(-A)^{1 / 2} \eta_{\alpha, \beta}^{h}(t, x)\right|^{2} \mathrm{~d} t\right)^{\gamma}\left(\int_{0}^{T}\left|\eta_{\alpha, \beta}^{h}(t, x)\right|^{2} \mathrm{~d} t\right)^{1-\gamma} .
\end{aligned}
$$

Now (3.17) follows from (3.19) and (3.20).

Corollary 3.4. Assume that Hypotheses $2.1,2.2$, and 2.3 hold. Let $\varphi \in C_{b}^{1}(H)^{5}$ and let $\alpha>0, \beta>0$. Then we have

$$
\left|D P_{t}^{\alpha, \beta} \varphi(x)\right| \leq \mathrm{e}^{-\omega t}\|\varphi\|_{1}, \quad x \in H
$$

Proof. Let Let $\varphi \in C_{b}^{1}(H), t \geq 0$ and $x \in H$. Then by (3.15) we have for any $h \in H$,

$$
\left\langle D P_{t}^{\alpha, \beta} \varphi(x), h\right\rangle=\mathbb{E}\left[\left\langle D \varphi\left(X_{\alpha, \beta}(t, x)\right), \eta_{\alpha, \beta}^{h}(t, x)\right\rangle\right],
$$

and so the conclusion follows from (3.19) and the arbitrariness of $h$.

We give now an infinite dimensional generalization of the Bismut-Elworthy formula $[1,15]$, which we shall use later. For this we need another assumption on $B$.

Hypothesis 3.5. We have Ker $B=\{0\}$. Moreover there is $\gamma \in[0,1)$ and a linear operator $V \in L(H)$ such that $B^{-1}=V A^{\gamma / 2}$.

Notice that Hypothesis 3.5 is obviously fulfilled in the Example 2.5, whereas it is fulfilled in the Example 2.6 provided $1 / 2<\delta<1$.

The following result was proved in [9] when $B=1$ and in [3] in the case of general reaction-diffusion systems.

\footnotetext{
${ }^{5} C_{b}^{1}(H)$ is the space of all mappings $\varphi: H \rightarrow \mathbb{R}$ which are uniformly continuous and bounded together with their first derivatives. For any $C_{b}^{1}(H)$ we set $\|\varphi\|_{1}=\|\varphi\|_{0}+\sup _{x \in H}|D \varphi(x)|$.
} 
Proposition 3.6. Assume that Hypotheses 2.1, 2.2, 2.3 and 3.5 hold. Let $\alpha>0, \beta>0$. Then for any $\varphi \in C_{b}(H)$ and any $t>0$ we have $P_{t}^{\alpha, \beta} \varphi \in C_{b}^{1}(H)$ and, for any $h \in H$,

$$
\left\langle D P_{t}^{\alpha, \beta} \varphi(x), h\right\rangle=\frac{1}{t} \mathbb{E}\left[\varphi\left(Y_{\alpha, \beta}(t, x)\right) \int_{0}^{t}\left\langle B^{-1} \eta_{\alpha, \beta}^{h}(s, x), \mathrm{d} W(s)\right\rangle\right], \quad x \in H,
$$

where $Y_{\alpha, \beta}(t, x)$ and $\eta_{\alpha, \beta}^{h}(s, x)$ are the mild solutions of (3.8) and (3.16) respectively.

Corollary 3.7. Assume that Hypotheses 2.1, 2.2, 2.3 and 3.5 hold. Then there exists a constant $k_{\gamma}>0$ such that for any $\varphi \in C_{b}(H), t>0, \alpha>0, \beta>0$ and for any $h \in H$, we have

$$
\left|D P_{t}^{\alpha, \beta} \varphi(x)\right| \leq k_{\gamma} t^{-(1+\gamma) / 2} \mathrm{e}^{-\omega t}\|\varphi\|_{0}, \quad x \in H, t>0 .
$$

Proof. By (3.22), using the Hölder inequality, we have

$$
\left|\left\langle D P_{t}^{\alpha, \beta} \varphi(x), h\right\rangle\right|^{2} \leq \frac{1}{t^{2}}\|\varphi\|_{0}^{2}\|V\|^{2} \int_{0}^{t} \mathbb{E}\left|(-A)^{-\gamma / 2} \eta_{\alpha, \beta}^{h}(s, x)\right|^{2} \mathrm{~d} s .
$$

Now, by Proposition 3.3 it follows that

$$
\left|\left\langle D P_{t}^{\alpha, \beta} \varphi(x), h\right\rangle\right|^{2} \leq \frac{2^{-\gamma}}{t^{1+\gamma}}\|V\|^{2}\|\varphi\|_{0}^{2}|h|^{2}
$$

and so, by the arbitrariness of $h$, we find

$$
\left|D P_{t}^{\alpha, \beta} \varphi(x)\right| \leq 2^{-\gamma / 2} t^{-(1+\gamma) / 2}\|V\|^{2}\|\| \varphi \|_{0}^{2}, \quad x \in H, t>0 .
$$

Now let $t \geq \varepsilon>0$. Then $D P_{t}^{\alpha, \beta} \varphi=D P_{t-\varepsilon}^{\alpha, \beta} P_{\varepsilon}^{\alpha, \beta} \varphi$. By Proposition 3.6 we have $P_{\varepsilon}^{\alpha, \beta} \in C_{b}^{1}(H)$, so that, in view of $(3.21)$,

Finally, by (3.25),

$$
\left|D P_{t}^{\alpha, \beta} \varphi(x)\right| \leq \mathrm{e}^{-\omega(t-\varepsilon)}\left\|P_{\varepsilon}^{\alpha, \beta} \varphi\right\|_{1}, \quad x \in H t \geq \varepsilon .
$$

$$
\left|D P_{t}^{\alpha, \beta} \varphi(x)\right| \leq 2^{-\gamma / 2} \varepsilon^{-(1+\gamma) / 2} \mathrm{e}^{-\omega(t-\varepsilon)}\|V\|^{2}\|\| \varphi \|_{0}, \quad x \in H, t \geq \varepsilon
$$

The conclusion follows by (3.25), and (3.26).

\subsection{A perturbation result}

Here we assume that Hypotheses 2.1, 2.2, 2.3 and 3.5 hold and that $G$ is Lipschitz continuous and bounded. Moreover we fix $\alpha>0, \beta>0$ and $\varphi \in C_{b}(H)$

The goal of this subsection is to obtain an estimate for $D T_{t}^{\alpha, \beta} \varphi$ independent of $\alpha$ and $\beta$, similar to the estimate (3.23) proved in Corollary 3.7 (where is essential the factor $\mathrm{e}^{-\omega t}$ ). This estimate will be needed to prove (1.5), see next section.

To this purpose we cannot follow the method used to prove Corollary 3.7. Assume in fact, to simplify, that $G \in C_{b}^{1}(H ; H)$. Then, by proceeding as before we arrive at the following estimate

$$
\left|D T_{t}^{\alpha, \beta} \varphi(x)\right| \leq k_{\gamma} t^{-(1+\gamma) / 2} \mathrm{e}^{\left(\|F\|_{1}-\omega\right) t}\|\varphi\|_{0}, \quad \varphi \in C_{b}(H), x \in H, t \geq 0
$$

If $\|F\|_{1}-\omega>0$ we cannot conclude that $\left|D T_{t}^{\alpha, \beta} \varphi(x)\right|$ vanishes as $t \rightarrow+\infty$, which is essential to prove (1.5). 
For this reason we use a different method by considering the following Kolmogorov equation (that was introduced in a different setting in [11]).

$$
\left\{\begin{array}{l}
D_{t} v_{\alpha, \beta}(t, x)=N_{\alpha, \beta} v_{\alpha, \beta}(t, x)+\left\langle G(x), D v_{\alpha, \beta}(t, x)\right\rangle, \quad t>0, x \in H, \\
v_{\alpha, \beta}(0, x)=\varphi(x), \quad \varphi \in C_{b}(H), x \in H,
\end{array}\right.
$$

which can be written in the following integral form

$$
v_{\alpha, \beta}(t, \cdot)=P_{t}^{\alpha, \beta} \varphi+\int_{0}^{t} P_{t-s}^{\alpha, \beta}\left(\left\langle G(\cdot), D v_{\alpha, \beta}(s, \cdot)\right\rangle\right) \mathrm{d} s .
$$

We shall solve equation (3.29) by a fixed point argument $\mathrm{n}$ the space $Z_{T}$ consisting of the set of all mappings $u:[0, T] \times H \rightarrow \mathbb{R}$ such that

(i) $u \in B_{b}([0, T] \times H)^{6}$.

(ii) $u(t, \cdot) \in C_{b}^{1}(H)$ for all $t>0$. (iii) $\sup _{t \in(0, T]} t^{(1+\gamma) / 2}\|u(t, \cdot)\|_{1}<+\infty$.

(iv) for all $x \in H, D u(\cdot, x)$ is measurable.

Then we shall show that

$$
v_{\alpha, \beta}(t, x)=T_{t}^{\alpha, \beta} \varphi(x), \quad x \in H, t \geq 0 .
$$

It is easy to check that $Z_{T}$, endowed with the norm

$$
\|u\|_{Z_{T}}:=\|u\|_{0}+\sup _{t \in(0, T]} t^{(1+\gamma) / 2}\|u(t, \cdot)\|_{1},
$$

is a Banach space.

Proposition 3.8. Assume that Hypotheses 2.1, 2.2, 2.3 and 3.5 hold and that $G$ is Lipschitz continuous and bounded. Then for any $\varphi \in C_{b}(H)$ there is a unique solution $v_{\alpha, \beta} \in Z_{T}$ of equation (3.29). Moreover

$$
v_{\alpha, \beta}(t, x)=T_{t}^{\alpha, \beta} \varphi(x), \quad x \in H, t \geq 0 .
$$

Proof. We write equation (3.29) as

where

$$
v_{\alpha, \beta}=P_{t}^{\alpha, \beta} \varphi+\gamma\left(v_{\alpha, \beta}\right),
$$

$$
\gamma(v)(t, \cdot)=\int_{0}^{t} P_{t-s}^{\alpha, \beta}(\langle G(\cdot), D v(s, \cdot)\rangle) \mathrm{d} s, t \geq 0, v \in Z_{T} .
$$

We first notice that $P_{t}^{\alpha, \beta} \varphi$ belongs to $Z_{T}$ in view of (3.22). Now we are going to show that $\gamma$ is a contraction on $Z_{T}$, provided $T$ is sufficiently small. Then the conclusion will follow by a standard fixed point argument. We have in fact

$$
|\gamma(v)(t, x)| \leq\|G\|_{0} \int_{0}^{t}|D v(s, \cdot)(x)| \mathrm{d} s \leq 2 t^{(1+\gamma) / 2}\|v\|_{Z_{T}}, \quad x \in H, t \geq 0,
$$

\footnotetext{
${ }^{6} B_{b}([0, T] \times H)$ is the space of all mappings $u:[0, T] \times H \rightarrow \mathbb{R}$ which are Borel and bounded.
} 
and, since

$$
D \gamma(v)(t, x)=\int_{0}^{t} D\left[P_{t-s}^{\alpha, \beta}(\langle G(\cdot), D v(s, \cdot)(x)\rangle)\right] \mathrm{d} s, \quad x \in H, t \geq 0
$$

we have

$$
\begin{aligned}
& t^{(1+\gamma) / 2}|D \gamma(v)(t, x)| \leq t^{(1+\gamma) / 2} \int_{0}^{t} \| D\left[P_{t-s}^{\alpha, \beta}(\langle G(\cdot), D v(s, \cdot)\rangle) \|_{0} \mathrm{~d} s\right. \\
& \leq t^{(1+\gamma) / 2}\|G\|_{0} \int_{0}^{t}(t-s)^{-(1+\gamma) / 2} s^{-(1+\gamma) / 2} \mathrm{~d} s\|v\|_{Z_{T}} \\
& =t^{(1+\gamma) / 2}\|G\|_{0} \int_{0}^{1}(1-s)^{-(1+\gamma) / 2} s^{-(1+\gamma) / 2} \mathrm{~d} s\|v\|_{Z_{T}} .
\end{aligned}
$$

Thus $\gamma$ maps $Z_{T}$ into $Z_{T}$ and it is a contraction, provided $T$ is sufficiently small, as required.

The last statement follows by a standard argument, see [7].

Corollary 3.9. Assume that Hypotheses 2.1, 2.2, 2.3 and 3.5 hold and that $G$ is Lipschitz continuous and bounded. Then there exists $c_{1, \gamma}>0$ such that for any $\varphi \in C_{b}(H)$ and any $t>0$ we have $T_{t}^{\alpha, \beta} \varphi \in C_{b}^{1}(H)$ and

$$
\left|D T_{t}^{\alpha, \beta} \varphi(x)\right| \leq c_{1, \gamma} t^{-(1+\gamma) / 2} \mathrm{e}^{-\omega t}\|\varphi\|_{0}, \quad x \in H, t>0
$$

Proof. Let $\varphi \in C_{b}(H)$, then by $(3.29)$ we have

$$
D T_{t}^{\alpha, \beta} \varphi=D P_{t}^{\alpha, \beta} \varphi+\int_{0}^{t} D P_{t-s}^{\alpha, \beta}\left(\left\langle G(\cdot), D T_{s}^{\alpha, \beta} \varphi\right\rangle\right) \mathrm{d} s .
$$

Taking into account (3.23) it follows that for any $x \in H$

$$
\left|D T_{t}^{\alpha, \beta} \varphi(x)\right| \leq \kappa_{\gamma} t^{-(1+\gamma) / 2} \mathrm{e}^{-\omega t}\|\varphi\|_{0}+\mathrm{d} s \kappa_{\gamma}\|G\|_{0} \int_{0}^{t} \mathrm{e}^{-\omega(t-s)}(t-s)^{-(1+\gamma) / 2}\left\|D T_{s}^{\alpha, \beta} \varphi\right\|_{0} \mathrm{~d} s .
$$

Then, setting

$$
g(t)=\mathrm{e}^{\omega t}\left\|D T_{t}^{\alpha, \beta} \varphi\right\|_{0}, \quad t \geq 0
$$

we have

$$
g(t) \leq \kappa_{\gamma} t^{-(1+\gamma) / 2}\|\varphi\|_{0}+\kappa_{\gamma}\|G\|_{0} \int_{0}^{t}(t-s)^{-(1+\gamma) / 2} g(s) \mathrm{d} s .
$$

By a well known generalization of the Gronwall lemma there exists $c_{\gamma}>0$ such that

$$
g(t) \leq c t^{-(1+\gamma) / 2}, \quad t>0
$$

Thus the conclusion follows.

Proposition 3.10. Assume that Hypotheses 2.1, 2.2, 2.3 and 3.5 hold and that $G$ is Lipschitz continuous and bounded. Then there exists $c>0$ such that for any $x, y \in H$, and any $\varphi \in C_{b}(H)$ we have

$$
\left|T_{t} \varphi(x)-T_{t} \varphi(y)\right| \leq c t^{-(1+\gamma)} \mathrm{e}^{-\omega t}\|\varphi\|_{0}|x-y|
$$


Proof. By (3.13) it follows that

$$
\lim _{\alpha, \beta \rightarrow 0} T_{t}^{\alpha, \beta} \varphi(x)=T_{t} \varphi(x), \varphi \in C_{b}(H), x \in H
$$

On the other hand, by (3.31) it follows that for any $x, y \in H$, and any $\varphi \in C_{b}(H)$ we have

$$
\left|T_{t}^{\alpha, \beta} \varphi(x)-T_{t}^{\alpha, \beta} \varphi(y)\right| \leq c t^{-(1+\gamma)} \mathrm{e}^{-\omega t}\|\varphi\|_{0}|x-y|
$$

Therefore the conclusion follows letting $\alpha$ and $\beta$ tend to zero.

Remark 3.11. By Proposition 3.10 it follows that $T_{t}$ is strong Feller. This result was proved by Cerrai [4] for general reaction-diffusion systems.

\subsection{Asymptotic behaviour of $\boldsymbol{T}_{\boldsymbol{t}}$}

We can prove now the main result of the paper.

Theorem 3.12. Assume that Hypotheses 2.1, 2.2, 2.3, and 3.5, hold and that $G$ is Lipschitz continuous and bounded. Then there exists $\kappa>0$ such that for any $\varphi \in C_{b}(H)$ we have

$$
\left|T_{t} \varphi(x)-\int_{H} \varphi(y) \zeta(\mathrm{d} y)\right| \leq \kappa t^{-(1+\gamma) / 2} \mathrm{e}^{-\omega t}(1+|x|)\|\varphi\|_{0}, \quad x \in H
$$

Moreover $\zeta$ is the unique invariant measure for $T_{t}$.

Proof. Let $\varphi \in C_{b}(H)$. Then, taking into account the invariance of $\zeta$, we obtain

$$
\left|T_{t} \varphi(x)-\int_{H} \varphi(y) \zeta(\mathrm{d} y)\right|=\left|\int_{H}\left[T_{t} \varphi(x)-T_{t} \varphi(y)\right] \zeta(\mathrm{d} y)\right| .
$$

Now by (3.32) we find

$$
\left|T_{t} \varphi(x)-\int_{H} \varphi(y) \zeta(\mathrm{d} y)\right| \leq c t^{-(1+\gamma) / 2} \mathrm{e}^{-\omega t} \int_{H}|x-y| \zeta(\mathrm{d} y)\|\varphi\|_{0} .
$$

But

$$
\int_{H}|x-y| \zeta(\mathrm{d} y) \leq|x|+\int_{H}|y| \zeta(\mathrm{d} y)<+\infty
$$

in virtue of (3.5). Therefore (3.33) is proved.

Finally, let $\eta$ be another invariant measure for $T_{t}$. Then by (3.33) we find,

$$
\lim _{t \rightarrow+\infty} \int_{H} T_{t} \varphi(x) \eta(\mathrm{d} x)=\int_{H} \varphi(x) d \eta(\mathrm{d} x)=\int_{H} \varphi(x) d \zeta(\mathrm{d} x)
$$

so that $\eta=\zeta$. 


\section{Application to Hamilton-Jacobi equations}

We are here concerned with the following Hamilton-Jacobi equation

$$
\left\{\begin{array}{l}
D_{t} u=\frac{1}{2} \operatorname{Tr}\left[B B^{*} D^{2} u\right]+\langle A x+F(x)+G(x), D u\rangle-\frac{1}{2}\left|B^{*} D u\right|^{2}, x \in D(A) \\
u(0)=\varphi \in C_{b}(H)
\end{array}\right.
$$

where $A, B, F$ and $G$ fulfill Hypotheses 2.1, 2.2, 2.3, and 3.5 and $G$ is Lipschitz continuous and bounded.

Equation (4.1) is related with the following optimal control problem:

minimize

$$
J(x, z):=\mathbb{E}\left(\int_{0}^{T}\left[\frac{1}{2}|z(t)|^{2}\right] \mathrm{d} t+\varphi(X(T, x ; z))\right),
$$

over all $z \in L_{W}^{2}\left(0, T ; L^{2}(\Omega, H)\right.$ ) (the Hilbert space of all square integrable processes adapted to $W$ defined on $[0, T]$ and with values in $H)$, subject to the state equation

$$
\left\{\begin{array}{l}
\mathrm{d} X=(A X+F(X)+G(X)+z(t)) \mathrm{d} t+B \mathrm{~d} W_{t}, t \geq 0 \\
X(0)=x \in H
\end{array}\right.
$$

In fact, as proved in [5], if $\varphi \in C_{b}^{1}(H)$ and $T>0$, equation (4.1) has a mild solution $u$ and there is a unique optimal control $z^{*}$ related to the optimal state $X^{*}$ by the feedback formula

$$
z^{*}(t)=-D u\left(T-t, X^{*}(t, x)\right), t \in[0, T]
$$

where $X^{*}$ is the solution of the closed loop equation

$$
\left\{\begin{array}{l}
\mathrm{d} X=(A X+F(X)+G(X)-D u(T-t, X)) \mathrm{d} t+Q^{1 / 2} \mathrm{~d} W(t), t \in[0, T] \\
X(0)=x \in H
\end{array}\right.
$$

Finally, the optimal cost is given by

$$
J^{*}(x)=u(T, x), \quad x \in H
$$

In [6] the control problem with infinite horizon but with a discount factor in the cost functional, is also solved.

In this section we want to show the existence of the limit of the solution $u(t, x)$ as $t \rightarrow+\infty$. This result can be used to study the infinite horizon problem without discount factor.

First we find an explicit solution of (4.1) by exploiting the special form of the Hamiltonian and using the well known Hopf transform. We notice that, in different situations, this method was used in [13]..

Namely we set $u(t, x)=-\log v(t, x), t \geq 0, x \in H$, so that (4.1) becomes

$$
\left\{\begin{array}{l}
D_{t} v=\frac{1}{2} \operatorname{Tr}\left[B B^{*} D^{2} u\right]+\langle A x+F(x)+G(x), D u\rangle, x \in D(A) \\
v(0)=\mathrm{e}^{-\varphi} .
\end{array}\right.
$$

Now the solution $v$ of (4.7) can be expressed in terms of the transition semigroup $T_{t}$ introduced in Section 3 as

$$
v(t, x)=T_{t}\left[\mathrm{e}^{-\varphi}\right](x), \quad t \geq 0, x \in H .
$$


Finally by Theorem 3.12 we find the following result:

Theorem 4.1. Assume that Hypotheses 2.1, 2.2, 2.3, and 3.5 hold and that $G$ is Lipschitz continuous and bounded. Let $u(t, x)$ be the solution of the Hamilton-Jacobi equation (4.1). Then for any $\varphi \in C_{b}(H)$ we have

$$
\lim _{t \rightarrow+\infty} u(t, x)=-\log \int_{H} \mathrm{e}^{-\varphi(y)} \zeta(\mathrm{d} y), \quad x \in H
$$

where $\zeta$ is the unique invariant measure for $T_{t}$.

\section{REFERENCES}

[1] J.M. Bismut, Large deviations and the Malliavin Calculus. Birkhäuser (1984).

[2] H. Brézis, Opérateurs maximaux monotones. North-Holland, Amsterdam (1973).

[3] S. Cerrai, A Hille-Yosida theorem for weakly continuous semigroups. Semigroup Forum 49 (1994) 349-367.

[4] S. Cerrai, Second order PDE's in finite and infinite dimensions. A probabilistic approach. Springer, Lecture Notes in Math. 1762 (2001).

[5] S. Cerrai, Optimal control problems for stochastic reaction-diffusion systems with non Lipschitz coefficients. SIAM J. Control Optim. 39 (2001) 1779-1816.

[6] S. Cerrai, Stationary Hamilton-Jacobi equations in Hilbert spaces and applications to a stochastic optimal control problem. SIAM J. Control Optim. (to appear).

[7] G. Da Prato, Stochastic evolution equations by semigroups methods. Centre de Recerca Matematica, Barcelona, Quaderns 11 (1998).

[8] G. Da Prato, A. Debussche and B. Goldys, Invariant measures of non symmetric dissipative stochastic systems. Probab. Theor. Related Fields (to appear).

[9] G. Da Prato, D. Elworthy and J. Zabczyk, Strong Feller property for stochastic semilinear equations. Stochastic Anal. Appl. 13 (1995) 35-45.

[10] G. Da Prato and M. Röckner, Singular dissipative stochastic equations in Hilbert spaces, Preprint. S.N.S. Pisa (2001).

[11] G. Da Prato and J. Zabczyk, Stochastic equations in infinite dimensions. Cambridge University Press (1992).

[12] G. Da Prato and J. Zabczyk, Ergodicity for Infinite Dimensional Systems. Cambridge University Press, London Math. Soc. Lecture Notes 229 (1996).

[13] G. Da Prato and J. Zabczyk, Differentiability of the Feynman-Kac semigroup and a control application. Rend. Mat. Accad. Lincei. 8 (1997) 183-188.

[14] E.B. Dynkin, Markov Processes, Vol. I. Springer-Verlag (1965).

[15] K.D. Elworthy, Stochastic flows on Riemannian manifolds, edited by M.A. Pinsky and V. Wihstutz. Birkhäuser, Diffusion Processes and Related Problems in Analysis II (1992) 33-72.

[16] W.H. Fleming and H.M. Soner, Controlled Markov processes and viscosity solutions. Springer-Verlag (1993).

[17] T. Kato, Nonlinear semigroups and evolution equations. J. Math. Soc. Japan 10 (1967) 508-520.

[18] K.R. Parthasarathy, Probability measures on metric spaces. Academic Press (1967). 